\title{
Overexpression of SPARC correlates with poor prognosis in patients with cervical carcinoma and regulates cancer cell epithelial-mesenchymal transition
}

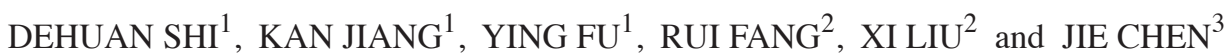 \\ ${ }^{1}$ Department of Obstetrics and Gynecology, Qilu Hospital; ${ }^{2}$ Grade 2011, Clinical Medicine, School of Medicine; \\ ${ }^{3}$ Department of Maternal and Child Health Care, School of Public Health, Shandong University, Jinan, \\ Shandong 250012, P.R. China
}

Received October 30, 2014; Accepted February 16, 2016

DOI: $10.3892 / \mathrm{ol} .2016 .4399$

\begin{abstract}
Secreted protein acidic and rich in cysteine (SPARC) is associated with the progression of numerous types of cancer. However, the role of SPARC in the progression of cervical cancer has not yet been adequately elucidated. In the current study, reverse transcription-quantitative polymerase chain reaction (RT-qPCR) and immunohistochemistry were employed to evaluate the mRNA and protein expression of SPARC in normal cervical tissue, cervical intraepithelial neoplasia (CIN) and cervical cancer. In addition, three epithelial-mesenchymal transition (EMT) markers (E-cadherin, $\mathrm{N}$-cadherin and vimentin) were detected by immunohistochemistry in the same specimens, and an enzyme-linked immunosorbent assay was conducted to detect the serum levels of SPARC in patients with cervical neoplasia. In highly invasive subclones of human cervical carcinoma cells, HeLa-1 and SiHa-1, lentiviral transfections were performed and RT-qPCR and western blot were used to investigate the effects of downregulated EGF-containing fibulin-like extracellular matrix protein 1 on the expression of E-cadherin, $\mathrm{N}$-cadherin and vimentin. The results revealed that, in cervical carcinoma tissue, SPARC expression was significantly upregulated in a manner that positively correlated with $\mathrm{N}$-cadherin and vimentin expression, and negatively correlated with E-cadherin expression. SPARC overexpression and high serum levels were significantly associated with the progression of cervical cancer and adverse prognosis of cervical cancer patients. Downregulation of SPARC can markedly reduce the expression of $\mathrm{N}$-cadherin and vimentin and increase the expression of E-cadherin. Thus, overexpression of SPARC is significantly
\end{abstract}

Correspondence to: Dr Dehuan Shi, Department of Obstetrics and Gynecology, Qilu Hospital, Shandong University, 107 Wenhua Xilu Road, Jinan, Shandong 250012, P.R. China

E-mail: shidehuan2014@163.com

Key words: SPARC, cervical carcinoma, prognosis, E-cadherin, $\mathrm{N}$-cadherin, vimentin associated with poor prognostic clinicopathological characteristics in cervical carcinoma, and may be important in EMT. The results of the current study suggest that SPARC may be a potential therapeutic option for individuals diagnosed with cervical carcinoma.

\section{Introduction}

Cervical cancer, a malignant neoplasm of the uterine cervix, is one of the most prevalent gynecological cancers worldwide. Although cervical cancer screening has been globally popularized, there are still large numbers of cases of advanced disease, the majority of which occur in the developing countries $(1,2)$. Therefore, studying the mechanisms of tumor invasion and metastasis, and possible methods of blocking these pathways, has the potential to greatly improve the prognosis of patients with cervical cancer.

Secreted protein acidic and rich in cysteine (SPARC) is a $43 \mathrm{kDa}$ protein that was first identified by Termine et al (3). As a calcium-binding matricellular glycoprotein, SPARC is able to interact with various extracellular matrix macromolecules and regulate cell adhesion, proliferation and migration (4). SPARC is overexpressed in the stroma and cancer cells in certain types of cancer, including breast cancer (5), melanoma (6), and glioma (7), affecting tumor development, invasion and metastasis. The importance of SPARC in the development of cancers and its potential role in cancer therapy have generated considerable interest in recent years $(8,9)$.

The epithelial-mesenchymal transition (EMT) is the process of transformation of polar epithelial cells into mesenchymal cells with the ability to invade and migrate. During this process, malignant cells disseminate from the primary epithelial neoplasm and invade the local tissue and blood vessels (10). EMT is closely associated with the invasion and metastasis of cancer cells (11). An important sign of EMT is the shift from the expression of E-cadherin to the expression of $\mathrm{N}$-cadherin, which facilitates the metastatic dissemination ability of malignant cells. Vimentin is a mesenchymal marker, and its upregulated expression is frequently associated with EMT (12).

In a previous study, compared with the low-invasiveness subclones, SPARC was found to be overexpressed in the highly 
invasive subclones. Additionally, knockdown of SPARC significantly inhibited cervical cancer cell proliferation, invasion and metastasis, accompanied by upregulated E-cadherin expression (13). Therefore, the current study aimed to investigate the expression of SPARC in cervical cancer specimens and assess the association between SPARC and the prognosis of cervical cancer patients, as well as to further study the role of SPARC in EMT.

\section{Materials and methods}

Cell lines. Human cervical cancer cells were cultured in Dulbecco's modified Eagle's medium (Gibco; Thermo Fisher Scientific, Inc., Waltham, MA, USA) supplemented with $10 \%$ fetal bovine serum (Gibco; Thermo Fisher Scientific, Inc.) at $37^{\circ} \mathrm{C}$ in a humidified atmosphere of $5 \% \mathrm{CO}_{2}$. Subclones of $\mathrm{HeLa}$ and SiHa human cervical carcinoma cells (Shanghai Institute for Biological Sciences, Chinese Academy of Sciences, Shanghai, China) were selected according to their differential invasiveness, as described in previous report (13).

RNA interference. The expression of SPARC was knocked down in cells using small hairpin RNAs (shRNAs; GeneChem, Co., Ltd., Shanghai, China), containing a cytomegalovirus-driven green fluorescent protein (GFP). The sequences for SPARC and the negative control were as follows: SPARC, 5'-AAC AAGACCTTCGACTCTTCC-3'; control, 5'-TTCTCCGAA CGTGTCACGT-3'. The invasive subclone cells, HeLa- 1 and SiHa-1, were seeded into six-well plates and then infected with lentiviral vector, which contained a multiple cloning site for insertion of shRNA constructs to be driven by an upstream U6 promoter (GeneChem, Co., Ltd.). In order to obtain the best transfection effect, varying concentration gradients were tested, and it was determined that one cell transfection required 60 viral units, which meant that the multiplicity of infection value was 60 . After $24 \mathrm{~h}$, fresh complete medium replaced the medium containing lentivirus. After another 4 days, $>80 \%$ GFP-positivity in the cells was observed using fluorescence microscopy, indicating successful transfection.

Tissue specimens. Patient specimens were obtained from the Department of Obstetrics and Gynecology of Shandong Provincial Hospital affiliated to Shandong University (Jinan, China) between June 2006 and June 2010. Patients were treated consecutively with conventional radiotherapy and chemotherapy, as follows: Paclitaxel (Bristol-Myers Squibb, New York, NY, USA) administered intravenously at a dose of $175 \mathrm{mg} / \mathrm{m}^{2}$ over a period of $3 \mathrm{~h}$ on day 1 of a 21 -day cycle, plus a carboplatin (Bristol-Myers Squibb) dose of $360 \mathrm{mg} / \mathrm{m}^{2}$, also administered intravenously on day 1 of the 21-day cycle, for 6 cycles. All patients received regular follow-up. During the study period, there were 9 patients who lost contact and 25 mortalities. The duration of follow-up was 2-7 years by the end of 2012. The study was approved by the Institutional Medical Ethics Committee of Shandong University.

Blood samples. All of the participants provided written informed consent indicating the willingness to donate their blood for research. Blood samples were collected in ethylenediaminetetraacetic acid (EDTA) tubes from the same
230 cervical cancer patients 60 cervical intraepithelial neoplasia (CIN) cases, 140 squamous cell carcinoma cases and 30 adenocarcinoma cases]. The EDTA-plasma samples from 40 healthy individuals were also obtained for comparison.

Enzyme-linked immunosorbent assay (ELISA). Sandwich ELISA (Human SPARC ELISA kit; \#LS-F12653; LifeSpan BioSciences, Inc., Seattle, WA, USA) was used to measure the levels of SPARC in different serum samples. Serum was diluted with enzyme immunoassay (EIA) buffer (containing $1 \%$ bovine serum albumin and $0.05 \%$ Tween 20 in phosphate buffer) from the ELISA kit and incubated for $2 \mathrm{~h}$ at $37^{\circ} \mathrm{C}$. After 4 washes with EIA buffer, mouse anti-human IgG horseradish peroxidase-conjugated antibodies (1:2,500 dilution) from the ELISA kit were added and incubated for $30 \mathrm{~min}$ at $4^{\circ} \mathrm{C}$. After a further 4 washes, $100 \mu \mathrm{l}$ tetramethylbenzidine solution (Sigma-Aldrich, St. Louis, MO, USA) was added and incubated for $30 \mathrm{~min}$ at room temperature. The reaction was subsequently stopped with $100 \mu 11 \mathrm{~N}$ sulfuric acid (Sigma-Aldrich). The experimental procedure was conducted according to the instruction manual and the results were measured using a SpectraMax ${ }^{\circledR}$ Plus 384 Microplate Reader (Molecular Devices, LLC, Sunnyvale, CA, USA) at $450 \mathrm{~nm}$.

Immunohistochemistry (IHC). IHC experiments were performed according to standard streptavidin-biotin-peroxidase complex procedures. Paraffin-embedded, $5-\mu \mathrm{m}$ thick sections were dewaxed in xylene, rehydrated in ethanol and fixed in 4\% paraformaldehyde for $30 \mathrm{~min}$. Antigen retrieval was then performed in $0.01 \mathrm{M}$ citrate buffer at $\mathrm{pH} 6.0$ for $15 \mathrm{~min}$ in a microwave oven. Paraffin, xylene, ethanol, paraformaldehyde and citrate buffer were all purchased from Sigma-Aldrich. The sections were incubated with goat polyclonal $\operatorname{IgG}$ anti-human SPARC (dilution, 1:500; \#AF941; R\&D Systems, Inc., Minneapolis, MN, USA), rabbit polyclonal IgG anti-human E-cadherin (dilution, 1:200; \#sc-7870; Santa Cruz Biotechnology, Inc., Dallas, TX, USA), goat polyclonal IgG anti-human N-cadherin (dilution, 1:200; \#sc-31031; Santa Cruz Biotechnology, Inc.) and goat polyclonal IgG anti-human vimentin (dilution, 1:200; \#sc-7557; Santa Cruz Biotechnology, Inc.) primary antibodies at $4^{\circ} \mathrm{C}$ overnight. Subsequently, sections were incubated for $1 \mathrm{~h}$ at room temperature with the following secondary antibodies: Mouse anti-goat IgG-B (dilution, 1:500; \#sc-2489; Santa Cruz Biotechnology, Inc.) for sections incubated with the goat polyclonal primary antibodies, and mouse anti-rabbit IgG-B (dilution, 1:500; \#sc-2491; Santa Cruz Biotechnology, Inc.) for sections incubated with the rabbit polyclonal primary antibody. and stained with the enzyme substrate 3',3-diaminobenzidine tetrahydrochloride (Sigma-Aldrich). Rabbit IgG (\#sc-2027; Santa Cruz Biotechnology, Inc.) was used as a negative control in the place of the primary antibody. An inverted microscope (IV953; Unico, Dayton, NJ, USA) was used to observe the sections. Brown granules in the cytoplasm or stroma were considered to be positive expression of SPARC.

IHC analysis. Immunohistochemical expression was evaluated using Image-Pro Plus 6.0 (Media Cybernetics, Inc., Rockville, MD, USA) to detect photodensity. In brief, 5 positive fields within a section were selected at random and read using Image-Pro Plus 6.0. The mean densities were subsequently 
Table I. Protein expression of SPARC in human cervical tissues.

\begin{tabular}{|c|c|c|c|c|c|c|c|}
\hline \multirow[b]{2}{*}{ Variables } & \multirow[b]{2}{*}{ Total } & \multicolumn{2}{|c|}{ SPARC low ${ }^{a}$} & \multicolumn{2}{|c|}{ SPARC high $^{\mathrm{b}}$} & \multirow[b]{2}{*}{$\chi^{2}$} & \multirow[b]{2}{*}{ P-value } \\
\hline & & $\mathrm{n}$ & $\%$ & $\mathrm{n}$ & $\%$ & & \\
\hline Normal & 40 & 39 & 97.5 & 1 & 2.5 & 88.9 & $<0.010$ \\
\hline Cervical intraepithelial neoplasia & 60 & 49 & 81.7 & 11 & 18.3 & & \\
\hline Carcinoma & 170 & 50 & 29.4 & 120 & 70.6 & & \\
\hline Pathology type & & & & & & 0.65 & 0.421 \\
\hline Squamous cell carcinoma & 140 & 43 & 30.7 & 97 & 69.3 & & \\
\hline Adenocarcinoma & 30 & 7 & 23.3 & 23 & 76.7 & & \\
\hline Cell differentiation & & & & & & 28.4 & $<0.010$ \\
\hline High and Medium & 89 & 42 & 47.2 & 47 & 52.8 & & \\
\hline Low & 81 & 8 & 9.9 & 73 & 90.1 & & \\
\hline Tumor stage & & & & & & 32.6 & $<0.010$ \\
\hline Stage I & 60 & 32 & 53.3 & 28 & 46.7 & & \\
\hline Stage II & 59 & 16 & 27.1 & 43 & 72.9 & & \\
\hline Stages III and IV & 51 & 2 & 3.9 & 49 & 96.1 & & \\
\hline Nodal status & & & & & & 28.3 & $<0.010$ \\
\hline Positive & 66 & 4 & 6.1 & 62 & 93.9 & & \\
\hline Negative & 104 & 46 & 44.2 & 58 & 55.8 & & \\
\hline
\end{tabular}

a Low, -/+; bhigh, ++/+++. SPARC, secreted protein acidic and rich in cysteine.
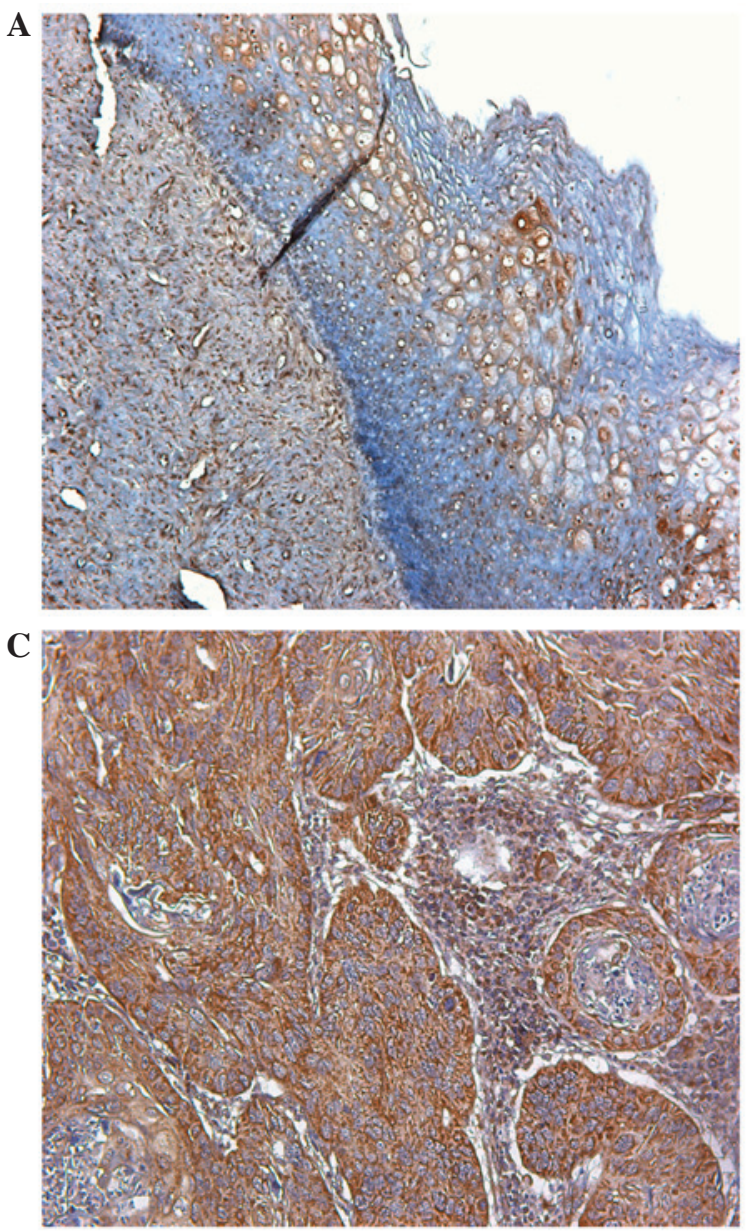

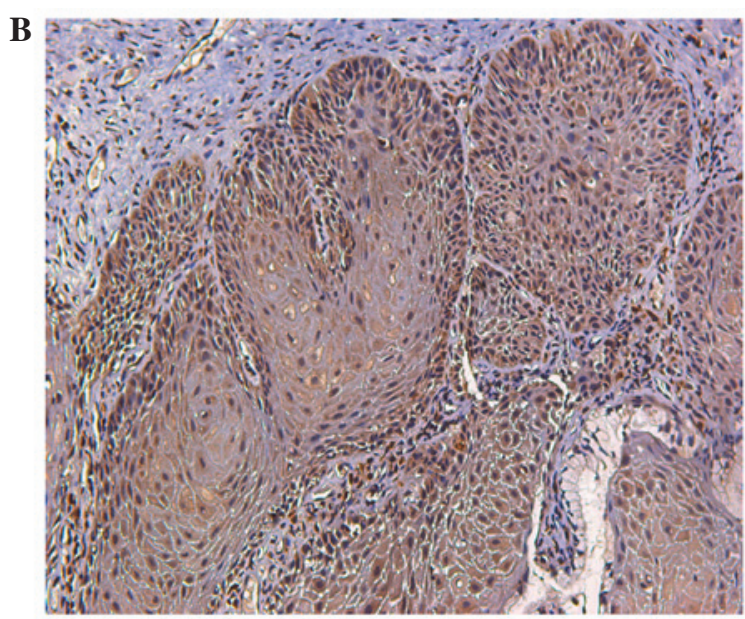

D

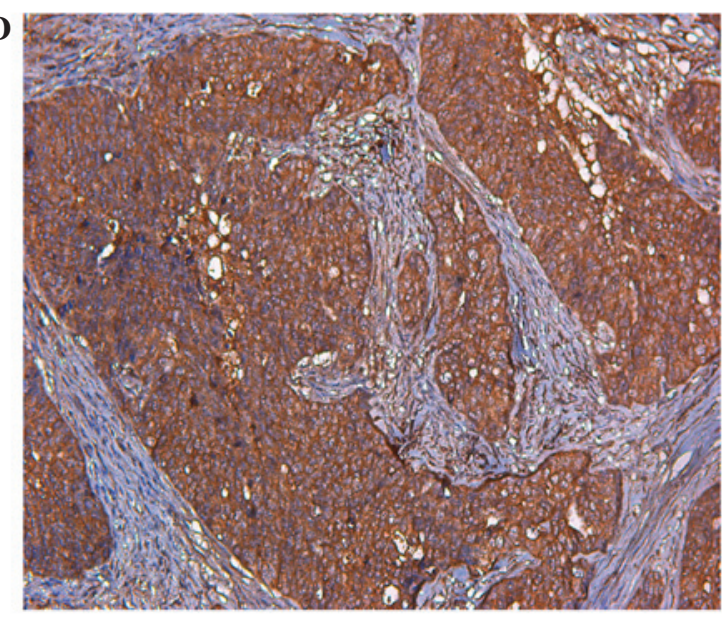

Figure 1. Expression of SPARC in human cervical tissues. (A) Normal human cervical tissue; (B) cervical intraepithelial neoplasia; (C) stage I-II cervical carcinoma; (D) stage III-IV cervical carcinoma (magnification, x200). 
Table II. mRNA expression of SPARC in human cervical tissues.

\begin{tabular}{|c|c|c|c|}
\hline & $\mathrm{n}$ & SPARC $^{\mathrm{a}}$ mRNA & P-value \\
\hline Control & 40 & $0.0087 \pm 0.0028$ & \\
\hline CIN & 60 & $0.0096 \pm 0.0034$ & $0.168^{b}$ \\
\hline Carcinoma & 170 & $0.0897 \pm 0.0103$ & $<0.010^{\mathrm{c}}$ \\
\hline Pathology type & & & 0.804 \\
\hline Squamous cell carcinoma & 140 & $0.0786 \pm 0.0157$ & \\
\hline Adenocarcinoma & 30 & $0.0994 \pm 0.0172$ & \\
\hline Cell differentiation & & & $<0.010$ \\
\hline High and medium & 89 & $0.0173 \pm 0.0083$ & \\
\hline Low & 81 & $0.0985 \pm 0.0117$ & \\
\hline Tumor stage & & & $<0.010$ \\
\hline Stage I & 76 & $0.0162 \pm 0.0076$ & \\
\hline Stage II & 81 & $0.0632 \pm 0.0094$ & \\
\hline Stages III and IV & 13 & $0.0983 \pm 0.0138$ & \\
\hline Nodal status & & & $<0.010$ \\
\hline Positive & 66 & $0.0986 \pm 0.0154$ & \\
\hline Negative & 104 & $0.0173 \pm 0.0067$ & \\
\hline
\end{tabular}

${ }^{\mathrm{a}}$ Mean \pm standard error; ${ }^{\mathrm{b}} \mathrm{CIN}$ vs. healthy control; ${ }^{\mathrm{c} C e r v i c a l}$ carcinoma vs. healthy control and CIN. SPARC, secreted protein acidic and rich in cysteine; CIN, cervical intraepithelial neoplasia.

Table III. Predictive factors of survival by multivariate analysis (Cox proportional hazards model).

\begin{tabular}{lcr}
\hline $\begin{array}{l}\text { Prognostic factor } \\
\begin{array}{l}\text { SPARC } \\
\text { (low vs. high) }\end{array}\end{array}$ & Hazard ratio (95\% CI) & P-value \\
$\begin{array}{l}\text { Pathology type } \\
\text { (SCC vs. ACA) }\end{array}$ & $0.978(0.429-2.230)$ & 0.957 \\
$\begin{array}{l}\text { Cell differentiation } \\
\text { (high + medium vs. low) }\end{array}$ & $0.999(0.998-1.000)$ & 0.075 \\
$\begin{array}{l}\text { Tumor stage } \\
\text { (stage III-IV vs. I-II) }\end{array}$ & $1.930(1.218-3.059)$ & 0.005 \\
$\begin{array}{l}\text { Lymph node metastasis } \\
\text { (positive vs. negative) }\end{array}$ & $1.019(1.009-1.029)$ & $<0.001$ \\
$\begin{array}{l}\text { Tumor size } \\
\text { Age }\end{array}$ & $1.001(0.996-1.005)$ & 0.771 \\
\hline
\end{tabular}

CI, confidence interval; SCC, squamous cell carcinoma; ACA, adenocarcinoma.

calculated. According to the intensity and percentage of positive staining, a semi-quantitative scoring system was used to evaluate SPARC expression (14). The staining intensity of SPARC was scored as 0 (negative), 1 (weak), 2 (moderate) or 3 (strong); while the percentage of positively stained cells was scored from 0 to 4 (score $0,0 \%$ cells stained; score $1,1-25 \%$; score 2, 26-50\%; score 3, 51-75\%; or score 4, 76-100\%). The final staining score (0-7) was calculated by adding together the intensity and percentage scores, and the scores of $0,1-3,4-5$, and 6-7 were converted into the sum indices,,-+++ and +++ ,

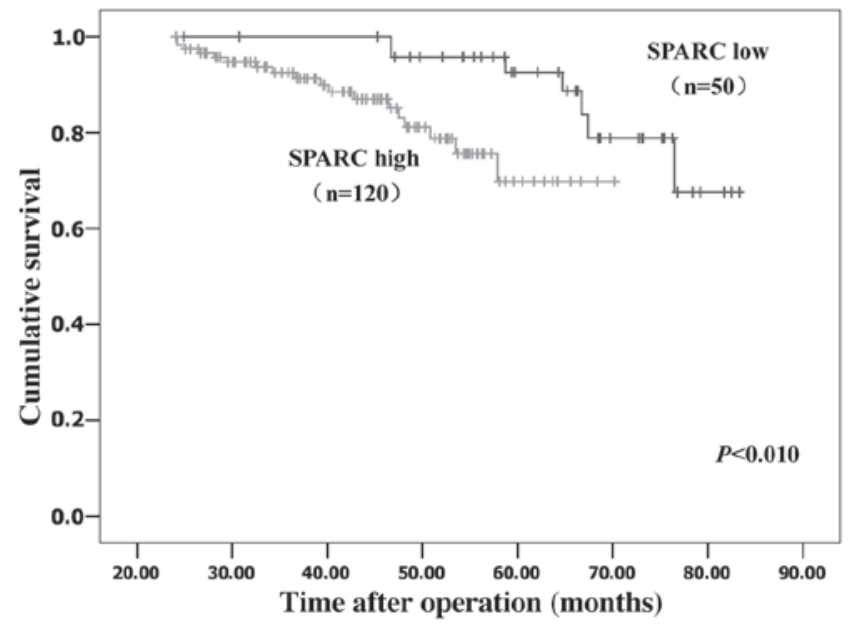

Figure 2. Kaplan-Meier analysis of the overall survival of patients with high or low tumor SPARC expression. The cohort included 170 patients with invasive cancer. Patients with high SPARC expression $(n=120)$ had a significantly poorer prognosis than those with low SPARC expression $(\mathrm{n}=50 ; \mathrm{P}<0.01)$. SPARC, secreted protein acidic and rich in cysteine.

respectively. For statistical analysis, low SPARC expression was defined as indices of - or + , while high SPARC expression was considered to be indicated by indices of ++ or +++ . Each tissue section was independently analyzed by three pathologists.

Reverse transcription (RT)-quantitative polymerase chain reaction ( $q P C R$ ). Total RNA was extracted using Invitrogen ${ }^{\mathrm{TM}}$ Trizol reagent (Thermo Fisher Scientific, Inc.) from human cervical cancer tissues and subclone cells. RNA was reverse transcribed into cDNA using the SuperScript ${ }^{\circledR}$ VILO $^{\mathrm{TM}}$ cDNA Synthesis kit (Thermo Fisher Scientific, Inc.) following the 

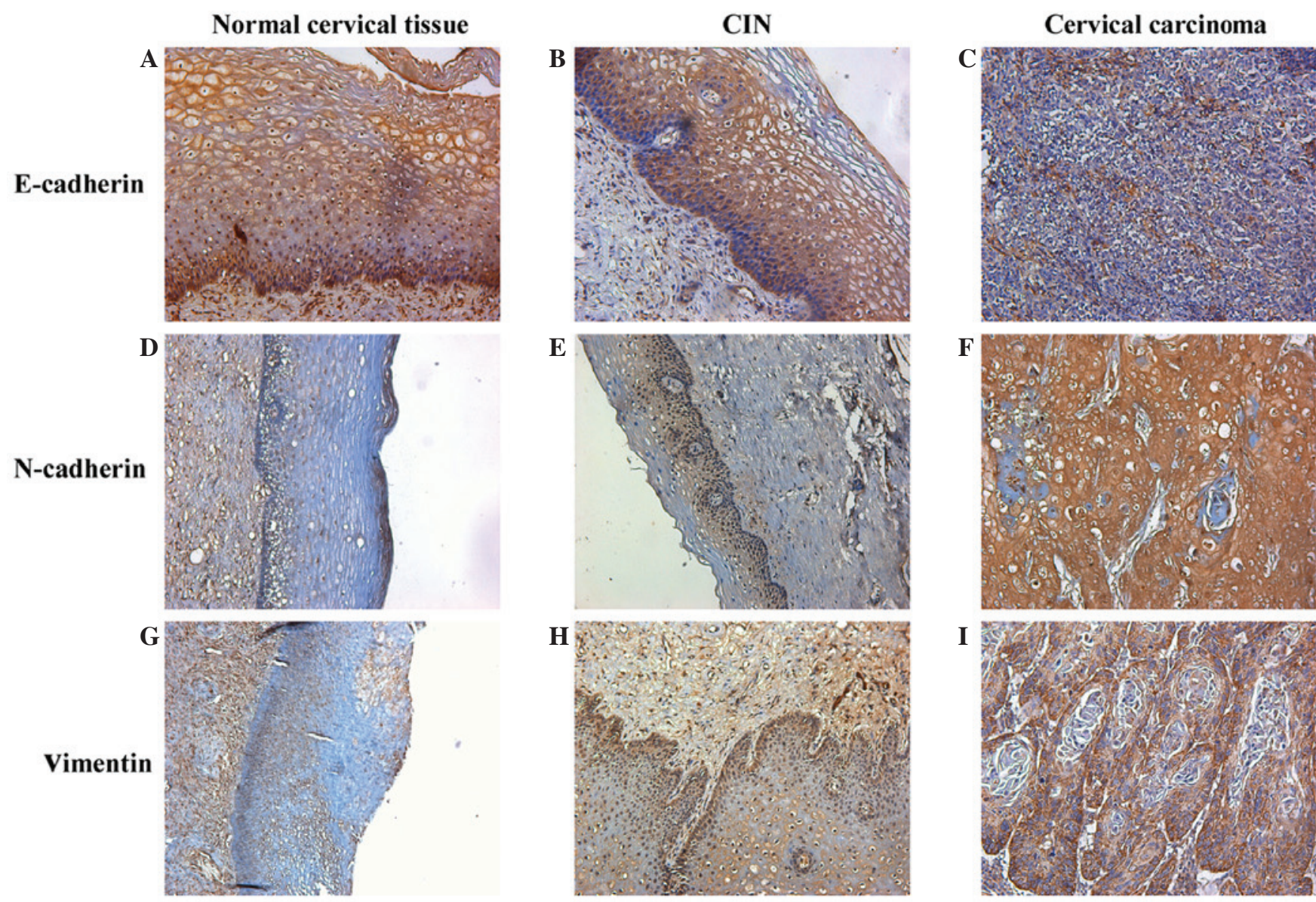

Figure 3. Immunohistochemical staining of E-cadherin, N-cadherin and vimentin. Immunohistochemical staining of E-cadherin in (A) normal human cervical tissue, (B) CIN and (C) cervical carcinoma (magnification, x200). Immunohistochemical staining of N-cadherin in (D) normal human cervical tissue (magnification, x100), (E) CIN (magnification, x100) and (F) cervical carcinoma (magnification, x200). Immunohistochemical staining of vimentin in (G) normal human cervical tissue (magnification, x100), (H) CIN (magnification, x200) and (I) cervical carcinoma (magnification, x200). CIN, cervical intraepithelial neoplasia.

manufacturer's protocols. The ABI PRISM 7500 Real-Time PCR System (Applied Biosystems Inc.; Thermo Fisher Scientific, Inc.) was used to perform qPCR analysis; a $20 \mu \mathrm{l}$ reaction volume contained $10 \mu 1$ Power SYBR Green PCR Master Mix (Applied Biosystems; Thermo Fisher Scientific, Inc.), $1 \mu \mathrm{l}$ of each primer ( $5 \mu \mathrm{mol} / \mathrm{l}), 1 \mu \mathrm{l}$ of cDNA template and $8 \mu \mathrm{l}$ DNAse/RNAse-free water (Sigma-Aldrich). The PCR cycle began with a denaturation step $\left(30 \mathrm{sec}\right.$ at $\left.95^{\circ} \mathrm{C}\right)$, followed by 40 repeated cycles of annealing/extension $(5 \mathrm{sec}$ at $95^{\circ} \mathrm{C}$ and $30 \mathrm{sec}$ at $60^{\circ} \mathrm{C}$. All reactions were performed in triplicate. Specific primers were designed by LightCycler ${ }^{\circledast}$ Probe Design software (Roche Diagnostics, Basel, Switzerland) and synthesized by Takara Biotechnology Co., Ltd. (Dalian, China). The sequences of primers were as follows: N-cadherin forward, 5'-GTGCCATCATTGCCA TCCTGCT-3', and reverse, 5'-CTGGTCTTCTTCTCCTCC ACCTTCT-3'; vimentin forward, 5'-GGAAGGCGAGGA GAGCAGGATT-3', and reverse, 5'-TTCAAGGTCATCGTG ATGCTGAGAAG-3'; E-cadherin forward, 5'-GGATTG CAAATTCCTGCCATTC-3', and reverse, 5'-AACGTTGTC CCGGGTGTCA-3'; SPARC forward, 5'-ACATAAGCCCAG TTCATCACCA-3', and reverse, 5'-ACAACCGATTCACCA ACTCCA-3'; $\beta$-actin forward, 5'-CCACGAAACTACCTT CAACTCCA-3', and reverse, 5'-GTGATCTCCTTCTGC
ATCCTGTC-3'. The $2^{-\Delta \Delta C q}$ method correlated with efficiency corrected normalized quantification results, relative quantification of gene expression was obtained by relative standard curves (15).

Western blot. Cells were washed twice with ice-cold phosphate-buffered saline and then lysed on ice in radioimmunoprecipitation assay buffer containing $1 \mathrm{mM}$ phenylmethylsulfonyl fluoride. Proteins (50 $\mu \mathrm{g} / \mathrm{lane})$ were resolved by sodium dodecyl sulfate polyacrylamide gel electrophoresis, transferred to polyvinyl difluoride membranes, and blocked with $5 \%$ bovine serum albumin. The membranes were first incubated with the aforementioned primary antibodies against SPARC, E-cadherin, $\mathrm{N}$-cadherin and vimentin at 1:1,000 dilutions overnight at $4^{\circ} \mathrm{C}$, and then incubated with bovine anti-goat IgG-AP (\#sc-2351) and bovine anti-rabbit IgG-AP (\#sc-2372) secondary antibodies (dilution, 1:1,000; Santa Cruz Biotechnology, Inc.) for $1 \mathrm{~h}$ at room temperature. Polyclonal goat IgG anti-human glyceraldehyde 3-phosphate dehydrogenase (dilution, 1:1,000; \#sc-20357; Santa Cruz Biotechnology, Inc.) was used as the control antibody incubated overnight at $4^{\circ} \mathrm{C}$. Blots were developed using the enhanced chemiluminescence method (Pierce ${ }^{\mathrm{TM}}$ ECL Western Blotting Substrate; Thermo Fisher Scientific, Inc.) 
Table IV. Serum levels of SPARC in patients with cervical tumors.

\begin{tabular}{|c|c|c|c|}
\hline & $\mathrm{n}$ & $\operatorname{SPARC}^{\mathrm{a}}(\mathrm{ng} / \mathrm{ml})$ & P-value \\
\hline Control & 40 & $124.73 \pm 76.28$ & \\
\hline CIN & 60 & $138.29 \pm 84.57$ & $0.416^{\mathrm{b}}$ \\
\hline Carcinoma & 170 & $486.58 \pm 135.84$ & $<0.010^{\mathrm{c}}$ \\
\hline Pathology type & & & 0.695 \\
\hline Squamous cell carcinoma & 140 & $463.74 \pm 136.22$ & \\
\hline Adenocarcinoma & 30 & $474.81 \pm 157.29$ & \\
\hline Cell differentiation & & & $<0.010$ \\
\hline High and medium & 89 & $143.61 \pm 83.64$ & \\
\hline Low & 81 & $496.73 \pm 132.78$ & \\
\hline Tumor stage & & & $<0.010$ \\
\hline Stage I & 76 & $107.16 \pm 65.34$ & \\
\hline Stage II & 81 & $243.19 \pm 96.35$ & \\
\hline Stages III and IV & 13 & $483.27 \pm 147.36$ & \\
\hline Nodal status & & & $<0.010$ \\
\hline Positive & 66 & $473.25 \pm 140.08$ & \\
\hline Negative & 104 & $132.91 \pm 86.59$ & \\
\hline
\end{tabular}

${ }^{\mathrm{a}}$ Mean \pm standard error; ${ }^{\mathrm{b}} \mathrm{CIN}$ vs. healthy control; ${ }^{\mathrm{c}}$ cervical carcinoma vs. healthy control and CIN. SPARC, secreted protein acidic and rich in cysteine; CIN, cervical intraepithelial neoplasia.

A

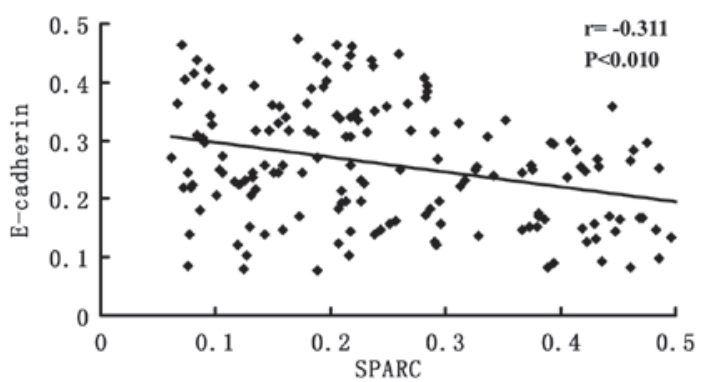

B

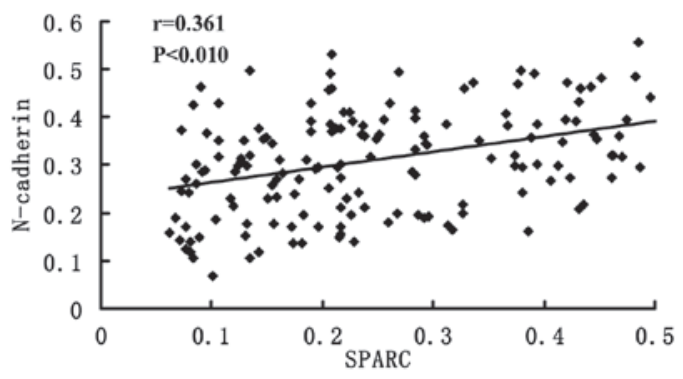

C

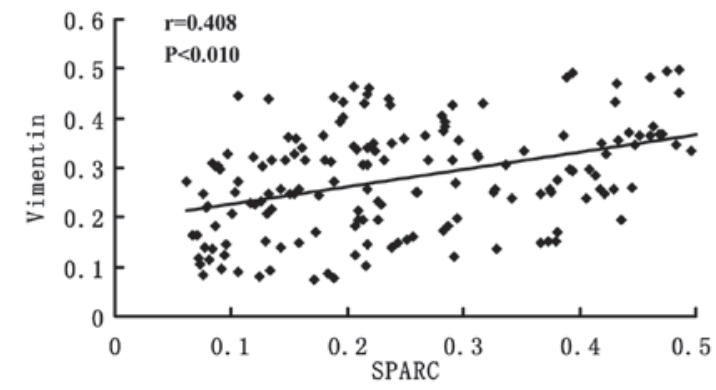

Figure 4. Pearson product-moment correlation coefficient analysis of SPARC with E-cadherin, $\mathrm{N}$-cadherin and vimentin expression. Using photodensities detected by software Image-Pro Plus 6.0, (A) expression of SPARC was negatively correlated with E-cadherin, and positively correlated with (B) N-cadherin and $(C)$ vimentin. SPARC, secreted protein acidic and rich in cysteine. and Gel-Pro 6.0 Analyzer Software (Media Cybernetics, Inc., Rockville, MD, USA) was used to analyze the intensity of the protein bands.

Statistical analysis. SPSS software version 13.0 (SPSS Inc., Chicago, IL, USA) was used for statistical analysis. A $\chi^{2}$ test was used to analyze IHC data. Measured data were recorded as the mean \pm standard error. A two-tailed t-test was used to compare the means between two sets, and a one-way analysis of variation was used to compare the means among three groups. Kaplan-Meier survival curves were calculated and analyzed using the log-rank test. Using Pearson's product-moment correlation coefficient, the associations between SPARC and E-cadherin, $\mathrm{N}$-cadherin and vimentin were analyzed. Individual prognosis was defined by multivariate Cox proportional hazard models. $\mathrm{P}<0.05$ (two-sided) was considered to indicate statistical significance.

\section{Results}

Expression of SPARC in human cervical cancer tissues. SPARC expression in normal human cervical tissue and CIN was very low compared with that in cervical carcinomas (Fig. 1A and B). High SPARC expression was detected in the cytoplasm of cancer cells and the stroma of cervical carcinomas (Fig. 1C and D). Furthermore, high SPARC expression was closely associated with poor differentiation, advanced stage and lymph node metastasis of cervical carcinomas (Table I). Similar results were also obtained through RT-qPCR; high SPARC mRNA expression was observed in cervical carcinoma, and was closely associated with their progression (Table II). 
A
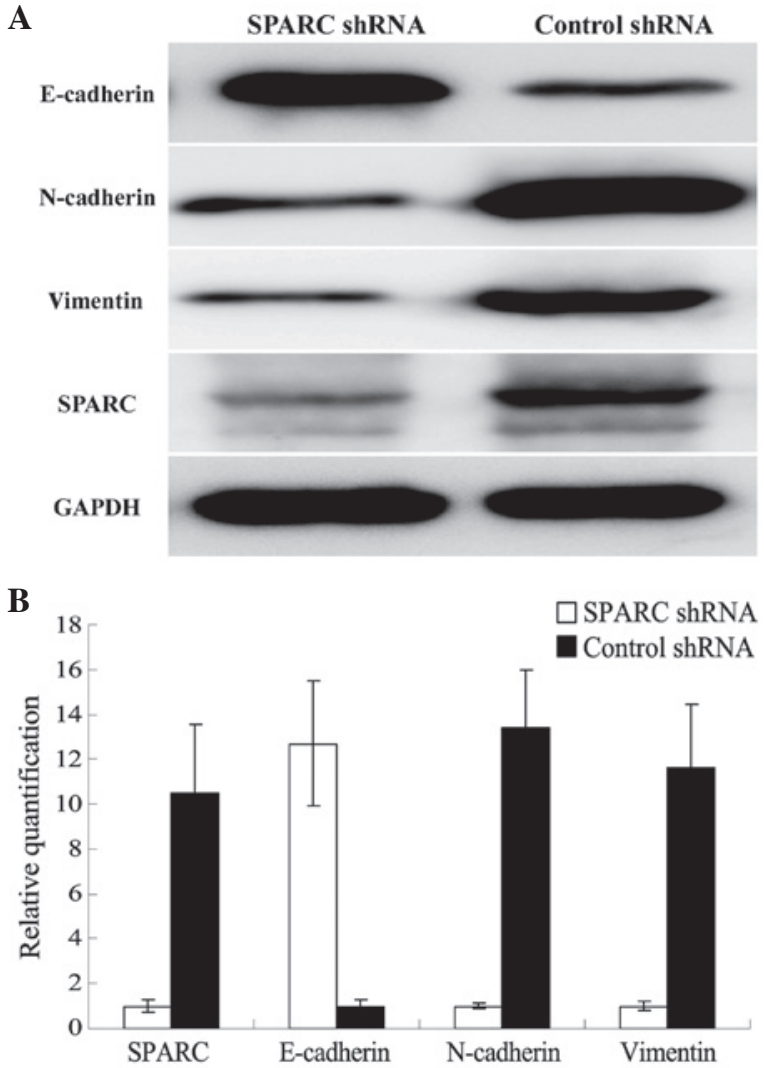

Figure 5. Effects of SPARC knockdown on the expression of E-cadherin, $\mathrm{N}$-cadherin and vimentin. (A) Western blot analysis measuring protein expression. The grey values of SPARC, E-cadherin, $\mathrm{N}$-cadherin and vimentin in SPARC shRNA-infected cells were $0.26 \pm 0.07,0.98 \pm 0.12,0.36 \pm 0.04$ and $0.31 \pm 0.06$ respectively, and the grey values of SPARC, E-cadherin, $\mathrm{N}$-cadherin and vimentin in control shRNA-infected cells were $0.95 \pm 0.09,0.32 \pm 0.03$, $0.97 \pm 0.11$ and $0.92 \pm 0.08$ respectively. There were significant differences between SPARC shRNA infected cells and control shRNA-infected cells $(\mathrm{P}<0.05)$. (B) Reverse transcription-quantitative polymerase chain reaction relative to ßactin measuring mRNA expression. SPARC, N-cadherin and vimentin mRNA expression in control shRNA-infected cells were 10.5 \pm 3.06 , $13.4 \pm 2.56$ and $11.6 \pm 2.85$ times than that in SPARC shRNA infected cells, however E-cadherin mRNA expression in SPARC shRNA infected cells was $12.7 \pm 2.79$ times than that in control shRNA-infected cells. There were significant differences between SPARC shRNA infected cells and control shRNA-infected cells $(\mathrm{P}<0.05)$.

Kaplan-Meier survival analyses were performed to evaluate the prognostic value of SPARC in cervical cancer. The results revealed that patients with low SPARC expression had a significantly more favorable prognosis than those with high SPARC expression (log rank, $\mathrm{P}<0.01$; Fig. 2). By multivariate analysis, considering all clinical and pathological factors together, lymph node metastasis $(\mathrm{P}<0.001$; hazard ratio, 1.019), expression of SPARC $(\mathrm{P}<0.001$; hazard ratio, 4.225) and stage of tumor $(\mathrm{P}=0.005$; hazard ratio, 1.930$)$ were significant prognostic factors (Table III).

Serum levels of SPARC in patients with cervical neoplasia and healthy controls. The mean serum levels of SPARC in healthy controls and CIN patients were markedly lower than that in patients with cervical carcinoma $(124.73 \pm 76.28$ and $138.29 \pm 84.57$ vs. $486.58 \pm 135.84 \mathrm{ng} / \mathrm{ml}$; $\mathrm{P}<0.010$; Table IV). There was no significant difference between healthy controls and CIN patients $(\mathrm{P}>0.05)$. Furthermore high SPARC serum levels were closely associated with poor differentiation, advanced stage and lymph node metastasis of cervical carcinomas $(\mathrm{P}<0.010)$. No significant differences were found between different pathological types of cervical cancer $(\mathrm{P}>0.05)$.

Associations between SPARC and EMT markers. Representative IHC staining images for E-cadherin, $\mathrm{N}$-cadherin and vimentin expressions are presented in Fig. 3. The photo densities of IHC staining images were evaluated by Image-Pro Plus 6.0. According to the Pearson product-moment correlation coefficient, the expression of E-cadherin and SPARC exhibited a negative correlation $(r=-0.311, \mathrm{P}<0.01$; Fig. $4 \mathrm{~A})$; however, the expression of $\mathrm{N}$-cadherin $(\mathrm{r}=0.361, \mathrm{P}<0.01$; Fig. 4B) and vimentin ( $r=0.408, \mathrm{P}<0.01$; Fig. $4 \mathrm{C})$ vs. SPARC exhibited strong positive correlations.

Effects of SPARC knockdown on expression of EMT markers. The expression levels of three EMT-related proteins (E-cadherin, $\mathrm{N}$-cadherin and vimentin) were detected by RT-qPCR and western blotting in SPARC shRNA-infected cells. As shown in Fig. 5, E-cadherin was upregulated and $\mathrm{N}$-cadherin and vimentin were downregulated following SPARC shRNA infection, in association with the knockdown of SPARC expression.

\section{Discussion}

To the best of our knowledge, the current study is the first to demonstrate that the expression of SPARC is significantly associated with poor clinicopathological characteristics and poor prognosis in human cervical carcinoma patients, and that it may have a role in the EMT of cervical cancer cells.

IHC analyses in the present study revealed that SPARC expression was significantly upregulated in cervical cancer tissues compared with normal cervical tissues and CIN. RT-qPCR experiments also confirmed that the mRNA expressions of SPARC was upregulated in cervical carcinoma tissues. Furthermore, high mRNA and protein expression levels of SPARC were associated with poor tissue differentiation, advanced stage and lymph node metastasis in cervical carcinomas. At present, few studies regarding SPARC and cervical cancer are available. A genome-wide screening study conducted by Sova et al in invasive cervical cancer showed that SPARC was upregulated in multiple cervical cancer cell lines and was aberrantly methylated; the aberrant methylation of SPARC was also observed at a high proportion in invasive cervical cancer clinical samples (16). In a previous study, we established highly invasive subclones and subclones with low invasiveness by the single cell cloning technology; subsequently, we identified that the expression of SPARC in the highly invasive subclones was much higher than that in those with low invasiveness. SPARC downregulation significantly suppressed cell proliferation, caused cell apoptosis, and inhibited cell invasion and metastasis in cervical cancer. Considering all of these findings together, we suggest that SPARC may promote the progression of cervical cancer.

SPARC is widely expressed in cancer and regulates cell survival, invasiveness and tumor-stroma interactions to promote tumor progression (17). One function of SPARC in carcinoma appears to be inhibiting the expression of E-cadherin and promoting EMT (8). In melanoma, it was demonstrated that 
SPARC could downregulate the expression of E-cadherin and $\mathrm{P}$-cadherin, induce the switch from E-cadherin to $\mathrm{N}$-cadherin, and enhance the expression of other extracellular proteins involved in EMT, which contributed to the dissemination of melanoma $(18,19)$. In lung cancer, ectopic expression of SPARC was observed to induce EMT with increased expression of vimentin and decreased expression of E-cadherin (20). EMT is important in the development of various types of epithelial cancer (21). Thus, the present study focused on assessing the association between SPARC and three EMT-related hallmarks, E-cadherin, $\mathrm{N}$-cadherin and vimentin, in cervical cancer. The results revealed that SPARC was inversely correlated with E-cadherin and positively correlated with $\mathrm{N}$-cadherin and vimentin; knockdown of SPARC resulted in increased expression of E-cadherin and reduced $\mathrm{N}$-cadherin and vimentin expression. SPARC may promote EMT-associated tumor invasion and contribute to cancer cell metastasis in cervical cancer.

In summary, the present study demonstrated that the expression of SPARC is associated with cancer cell invasion and metastasis, and poor prognosis in cervical cancer patients. We hypothesize that SPARC may be important during EMT. These observations support our belief that SPARC is a promising therapeutic target for the inhibition of metastasis and is a prognostic biomarker for cervical cancer.

\section{Acknowledgements}

This study was supported by the China Postdoctoral Science Foundation (no. 2014M551919). The funding organizations had no role in the experimental design, data collection and analysis, or the manuscript preparation.

\section{References}

1. Ferlay J, Shin HR, Bray F, Forman D, Mathers C and Parkin DM: Estimates of worldwide burden of cancer in 2008: GLOBOCAN 2008. Int J Cancer 127: 2893-2917, 2010.

2. Waggoner SE: Cervical cancer. Lancet 361: 2217-2225, 2003.

3. Termine JD, Kleinman HK, Whitson SW, Conn KM, McGarvey ML and Martin GR: Osteonectin, a bone-specific protein linking mineral to collagen. Cell 26: 99-105, 1981.

4. Bornstein P and Sage EH: Matricellular proteins: Extracellular modulators of cell function. Curr Opin Cell Biol 14: 608-616, 2002.

5. Hsiao YH, Lien HC, Hwa HL, Kuo WH, Chang KJ and Hsieh FJ: SPARC (osteonectin) in breast tumors of different histologic types and its role in the outcome of invasive ductal carcinoma. Breast J 16: 305-308, 2010.
6. Botti G, Scognamiglio G, Marra L, Collina F, Di Bonito M, Cerrone M, Grilli B, Anniciello A, Franco R, Fulciniti F, et al: SPARC/osteonectin is involved in metastatic process to the lung during melanoma progression. Virchows Arch 465: 331-338, 2014.

7. Seno T, Harada H, Kohno S, Teraoka M, Inoue A and Ohnishi T: Downregulation of SPARC expression inhibits cell migration and invasion in malignant gliomas. Int J Oncol 34: 707-715, 2009.

8. Feng J and Tang L: SPARC in Tumor Pathophysiology and as a Potential Therapeutic Target. Curr Pharm Des 20: 6182-6190, 2014.

9. Wang B, Chen K, Xu W, Chen D, Tang W and Xia TS: Integrative genomic analyses of secreted protein acidic and rich in cysteine and its role in cancer prediction. Mol Med Rep 10: 1461-1468, 2014.

10. Singh A and Settleman J: EMT, cancer stem cells and drug resistance: An emerging axis of evil in the war on cancer. Oncogene 29: 4741-4751, 2010.

11. Steinestel K, Eder S, Schrader AJ and Steinestel J: Clinical significance of epithelial-mesenchymal transition. Clin Transl Med 3: 17, 2014.

12. Thiery JP, Chua K, Sim WJ and Huang R: Epithelial mesenchymal transition during development in fibrosis and in the progression of carcinoma. Bull Cancer 97: 1285-1295, 2010 (In French).

13. Chen J, Shi D, Liu X, Fang S, Zhang J and Zhao Y: Targeting SPARC by lentivirus-mediated RNA interference inhibits cervical cancer cell growth and metastasis. BMC Cancer 12: 464, 2012.

14. Soumaoro LT, Uetake H, Higuchi T, Takagi Y, Enomoto M and Sugihara K: Cyclooxygenase-2 expression: A significant prognostic indicator for patients with colorectal cancer. Clin Cancer Res 10: 8465-8471, 2004.

15. Schmittgen TD, Zakrajsek BA, Mills AG, Gorn V, Singer MJ and Reed MW: Quantitative reverse transcription-polymerase chain reaction to study mRNA decay: Comparison of endpoint and real-time methods. Anal Biochem 285: 194-204, 2000.

16. Sova P, Feng Q, Geiss G, Wood T, Strauss R, Rudolf V, Lieber A and Kiviat N: Discovery of novel methylation biomarkers in cervical carcinoma by global demethylation and microarray analysis. Cancer Epidemiol Biomarkers Prev 15: 114-123, 2006.

17. Arnold SA and Brekken RA: SPARC: A matricellular regulator of tumorigenesis. J Cell Commun Signal 3: 255-273, 2009.

18. Girotti MR,Fernández M,López JA, Camafeita E, Fernández EA, Albar JP, Benedetti LG, Valacco MP, Brekken RA, Podhajcer OL and Llera AS: SPARC promotes cathepsin B-mediated melanoma invasiveness through a collagen I/ $\alpha 2 \beta 1$ integrin axis. J Invest Dermatol 131: 2438-2447, 2011.

19. Fenouille N, Tichet M, Dufies M, Pottier A, Mogha A, Soo JK, Rocchi S, Mallavialle A, Galibert MD, Khammari A, et al: The epithelial-mesenchymal transition (EMT) regulatory factor SLUG (SNAI2) is a downstream target of SPARC and AKT in promoting melanoma cell invasion. PLoS One 7: e40378, 2012.

20. Miao L, Wang Y, Xia H, Yao C, Cai H and Song Y: SPOCK1 is a novel transforming growth factor- $\beta$ target gene that regulates lung cancer cell epithelial-mesenchymal transition. Biochem Biophys Res Commun 440: 792-797, 2013.

21. Wendt MK, Balanis N, Carlin CR and Schiemann WP: STAT3 and epithelial-mesenchymal transitions in carcinomas. JAKSTAT 3: e28975, 2014. 